\title{
Unusual Case of Indolent Choroidal Alterations Mimicking Neurofibromatosis Type 1
}

\author{
Solmaz Abdolrahimzadeh ${ }^{a} \quad$ Martina Formisano $^{b} \quad$ Fabio Guglielmelli $^{a}$ \\ Stefano Amodeo ${ }^{a} \quad$ Maria Carmela Costa ${ }^{c}$ Gianluca Scuderi ${ }^{a}$ \\ aOphthalmology Unit, Neurosciences, Mental Health and Sensory Organs (NESMOS) \\ Department, Sapienza University of Rome, St. Andrea Hospital, Rome, Italy; \\ bOphthalmology Unit, Sapienza University of Rome, Department of Sense Organs, \\ Policlinico Umberto I, Rome, Italy; 'Ophthalmology Unit, Ospedale Vito Fazzi, Lecce, Italy
}

\section{Keywords}

Choroidal alterations $\cdot$ Near-infrared reflectance $\cdot$ Neurofibromatosis type $1 \cdot$ Choroidal imaging $\cdot$ Spectral-domain optical coherence tomography

\begin{abstract}
Indolent, non-progressive choroidal alterations can be strongly suggestive of neurofibromatosis type 1 (NF1) but are also rarely of unknown aetiology. A 63-year-old man presented for a routine examination. Comprehensive ophthalmological examination and retinal imaging was performed. Visual acuity was 20/20. The anterior segment and fundus were unremarkable. Near-infrared reflectance (NIR) with spectral-domain optical coherence tomography showed unilateral hyperreflective areas in the left posterior pole, corresponding to choroidal nodules on enhanced depth imaging and hypofluorescent areas on indocyanine green angiography. Dermatological evaluation and genetic testing for NF1 were negative. Chest computed tomography, liver function, HLA-A29, and angiotensin-converting enzyme level were negative. The patient has remained in good health and the choroidal alterations have remained non-progressive for 3 years. Choroidal alterations observed with NIR could be a manifestation of somatic mosaicism or a variation of a new unclassified correlated condition that may be better elucidated in the future, given the use of novel imaging techniques that are currently available in ophthalmology.




\section{Introduction}

Clinical entities with yellow or white multifocal alterations of the fundus can be due to the white dot syndrome, various choroidopathies, and multifocal posterior placoid pigment epitheliopathy with presumed autoimmune or viral aetiology. Other causes could be neoplastic and infectious disease such as lymphoma, uveal metastatic lesions, tuberculosis, or sarcoidosis. Nevertheless, these conditions usually present with a history of disease and symptomatology. Choroidal alterations are currently reported in up to $90 \%$ of patients with neurofibromatosis type 1 (NF1) with near-infrared reflectance (NIR) imaging that enables rapid and noninvasive evaluation of the choroid [1-4]. These lesions are undetectable by fundus examination or fluorescein angiography (FAG), but are visible with indocyanine green angiography (ICGA) [5].

There is a paucity of literature on indolent choroidal alterations observed in healthy subjects; two articles reported choroidal alterations in healthy subjects during screening for NF1 $[1,6]$, and a recent report described 4 patients with indolent, non-progressive choroidal alterations where aetiology could not be established [7].

The present paper is a report on a healthy patient in whom non-progressive indolent choroidal nodules, undetected with fundus examination, were observed with NIR. Although the aetiology could not be established, this report is aimed to highlight the role and importance of novel imaging techniques in the armamentarium of ophthalmologists.

\section{Case Presentation}

A healthy 63-year-old man presented to our clinic for routine ophthalmological examination. The history of the patient was positive for noninvasive bladder cancer in 2013, treated with transurethral resection of bladder tumour (TURBT), followed by intravesical therapy with Bacillus Calmette-Guérin. The patient underwent regular follow-ups with no recurrences. History was negative for any other systemic or ocular diseases and inflammation. Bestcorrected visual acuity was 20/20, and intraocular pressure was $12 \mathrm{~mm} \mathrm{Hg}$ in both eyes. Slitlamp examination was unremarkable, and no Lisch nodules were detected; the pupillary reflexes and intraocular pressure were normal. Indirect binocular fundus ophthalmoscopy was unremarkable, and there were no signs of vitreous inflammation in both eyes (Fig. 1). NIR images at $815 \mathrm{~nm}$ (Spectralis OCT Family Acquisition Module, V 5.1.6.0 Heidelberg Engineering) revealed rounded hyperreflective bright areas and irregular patchy hyperreflectivity prevalently of the posterior pole in the left eye (Fig. 2). The NIR images were used to evaluate the hyperreflective areas on enhanced depth imaging using spectral-domain optical coherence tomography cross-sections, which corresponded to "dome-shaped" and "placoid" hyperreflective nodules, typical of NF1 (Fig. 3). There were no alterations of the peripapillary retinal nerve fiber layer. FAG showed slight staining of the lesions in the late frames, and ICG-A showed multiple hypofluorescent areas in the late views, corresponding to the choroidal abnormalities (Fig. 4). Dermatological examination was negative, there were no family members with NF1, and there were no NF1 and SPRED1 gene mutations. Computed tomography of the chest, to rule out tuberculosis and sarcoidosis, was unremarkable. Comprehensive blood tests including liver function to exclude possible malignancy, HLA-A29, and angiotensin-converting enzyme, were negative. Orbital and bulbar B scan ultrasound ruled out orbital malignancy.

The patient has remained in good general health and the choroidal alterations have remained non-progressive for 3 years. 


\section{Case Reports in Ophthalmology}

\section{Discussion}

In this paper, we present the case of a healthy subject with unilateral multiple, indolent, non-progressive choroidal alterations with imaging characteristics strongly suggestive of NF1. However, diagnostic workup was negative for NF1, inflammatory, infective, neoplastic, and chorioretinal disease.

NIR images enable to study the choroid due to the passage of long-infrared light past melanin and lipofuscin [4]. NIR imaging in the case described herein revealed rounded hyperreflective bright areas and irregular patchy hyperreflectivity typical of NF1 in the left eye of the patient, these corresponded to "dome-shaped" and "placoid" hyperreflective choroidal nodules using enhanced depth imaging [2]. The choroidal alterations corresponded to hypofluorescent areas on ICG-A [5]. Based on the NIH Consensus Development Statement, diagnostic ophthalmic signs of NF1 include Lisch nodules of the iris surface in over $90 \%$ of the patients and optic pathway gliomas in $15-20 \%$ of children before the age of 8 years [8-10]. Parrozzani et al. [11] showed a feasibility rate of detecting choroidal nodules with NIR in NF1 at $82 \%$ with a sensitivity, specificity, and positive and negative predictive values of $0.60,0.97,0.98$, and 0.46 , respectively. They reported that choroidal abnormalities ranked third among the $\mathrm{NIH}$ criteria for positive predictive value [11]. Other ophthalmic characteristics of NF1 include microvascular retinal anomalies, choroidal vascular alterations, and retinal nerve fiber layer and ganglion cell layer-inner plexiform layer thinning [12-15]; however, the patient was negative for these signs. Only two articles in the literature, when screening for NF1, showed the presence of choroidal nodules in healthy subjects. Viola et al. [1] found nodules in $82 \%$ of 95 patients with NF1 and 7\% of 100 healthy patients. They, however, reported that these were due to atrophic areas, vitreoretinal reflex, and optical reflectance artefacts in 4 patients and choroidal nevi in 3 patients [1]. Indeed, choroidal nevi are detectable by fundus examination in the vast majority of cases and Vallabh et al. [16] described NIR imaging hyperreflectivity in a large cohort of patients. Goktas et al. [6] studied 19 patients with NF1 and 20 control subjects and found unilateral choroidal alterations in 1 healthy subject.

Genetic conditions may at times manifest with clinical features in a segmental form in patchy, circumscribed, or linear arrangements, or can be in organs or localised regions [17]. Somatic mosaicism has been suggested as the mechanism for the NF1 gene $[18,19]$. In the general population, the prevalence of segmental NF1 is estimated to be about $0.002 \%$ [20]. Segmental manifestations of NF1 can be limited to one segment, one quadrant, or one half of the body in a symmetrical or asymmetrical fashion [20,21]. Rare, incomplete, or unilateral ophthalmic manifestations have been described but, to our knowledge, cases of segmental NF1 with choroidal nodules have not been previously reported in the literature [20, 21]. Interestingly, in considering dermatological conditions, Cassiman et al. [22] have recently described the presence of choroidal nodules in Legius syndrome in $18 \%$ of 11 patients.

Indolent choroidal alterations have been described in a case of solitary choroidal tuberculoma in a patient with no other systemic signs of tuberculosis [23] and in one case of ocular sarcoidosis without any traceable signs at fundus examination despite hypofluorescence on ICG-A [24]. Interestingly, Shelsta et al. [7] reported four cases presenting with indolent, yellow, or white choroidal lesions visible with fundus examination of unknown aetiology that remained stable over time. These authors did not report optical coherence tomography changes in 3 of the patients, whereas enhanced depth imaging in one case was positive for dome-shaped hyporeflective choroidal alterations, contrasting with the hyperreflective dome-shaped choroidal nodules, typical of NF1, observed in the case reported herein [7]. 
NIR imaging increases the armamentarium of ophthalmologists in the diagnosis, classification, and eventual comprehension of physiological and pathological mechanisms in health and disease. It is reasonable to recommend the acquisition of NIR images of the posterior pole in all patients. NIR images are an adjunct to fundus examination, as they can provide additional information on the optic nerve head, retinal vasculature, and reflectivity alterations of the posterior pole.

\section{Conclusions}

The advent of NIR imaging enables noninvasive evaluation of the choroid, but alterations and their significance, or their association with dermatological diseases is still in an early phase. The absence of ophthalmoscopic fundus alterations, rounded hyperreflective bright areas, and irregular patchy hyperreflectivity shown with NIR, and the hyperreflective nature of the choroidal nodules evidenced by enhanced depth imaging induces us to hypothesise a possible case of somatic mosaicism or a variation of a new unclassified correlated condition.

\section{Statement of Ethics}

The patient gave written informed consent to publish the case and the study was carried out according to the tenets of the Declaration of Helsinki.

\section{Disclosure Statement}

The authors have no conflicts of interest to declare.

\section{Funding Sources}

No funding was received for this study.

\section{Author Contributions}

Prof. Abdolrahimzadeh took the lead in drafting, writing the manuscript, interpreting images, and performed critical reading for important intellectual content. Dr. Formisano drafted the manuscript, carried out imaging and patient management, and contributed to the writing of the final manuscript. Dr. Guglielmelli, Dr. Amodeo, and Dr. Costa contributed to manuscript drafting, literature search, and image evaluation. Prof. Scuderi drafted the manuscript, interpreted images, and performed critical reading for important intellectual content. All authors approved the final version of the manuscript. 


\section{References}

1 Viola F, Villani E, Natacci F, Selicorni A, Melloni G, Vezzola D, et al. Choroidal abnormalities detected by nearinfrared reflectance imaging as a new diagnostic criterion for neurofibromatosis 1. Ophthalmology. 2012 Feb;119(2):369-75.

2 Abdolrahimzadeh S, Felli L, Plateroti R, Plateroti AM, Giustini S, Calvieri S, et al. Morphologic and vasculature features of the choroid and associated choroid-retinal thickness alterations in neurofibromatosis type $1 . \mathrm{Br}$ ] Ophthalmol. 2015 Jun;99(6):789-93.

3 Abdolrahimzadeh S, Plateroti AM, Recupero SM, Lambiase A. An update on the ophthalmologic features in the phakomatoses. J Ophthalmol. 2016;2016:3043026.

4 Spaide RF, Koizumi H, Pozzoni MC. Enhanced depth imaging spectral-domain optical coherence tomography Am J Ophthalmol. 2008 0ct;146(4):496-500.

5 Yasunari T, Shiraki K, Hattori H, Miki T. Frequency of choroidal abnormalities in neurofibromatosis type 1. Lancet. 2000 Sep;356(9234):988-92.

6 Goktas S, Sakarya Y, Ozcimen M, Alpfidan I, Uzun M, Sakarya R, et al. Frequency of choroidal abnormalities in pediatric patients with neurofibromatosis type 1. J Pediatr Ophthalmol Strabismus. 2014 Jul;51(4):204-8.

7 Shelsta HN, Jampol LM, Weinberg DV. Indolent nonprogressive multifocal choroidal lesions. Retina. 2012 Feb;32(2):289-92.

8 National Institutes of Health Consensus Development Conference. Neurofibromatosis. Conference statement. Arch Neurol. 1988 May;45(5):575-8.

9 Recupero SM, Plateroti R, Abdolrahimzadeh S, De Dominicis M, Giustini S, Noviello MR, et al. Lisch nodules in neurofibromatosis type 1: relationship to age and cutaneous neurofibromas. Ann Ophthalmol-Glaucoma. 1996;28(3):178-83.

10 Parrozzani R, Clementi M, Kotsafti O, Miglionico G, Trevisson E, Orlando G, et al. Optical coherence tomography in the diagnosis of optic pathway gliomas. Invest Ophthalmol Vis Sci. 2013 Dec;54(13):8112-8.

11 Parrozzani R, Clementi M, Frizziero L, Miglionico G, Perrini P, Cavarzeran F, et al. In vivo detection of choroidal abnormalities related to NF1: feasibility and comparison with standard NIH diagnostic criteria in pediatric patients. Invest Ophthalmol Vis Sci. 2015 Sep;56(10):6036-42.

12 Abdolrahimzadeh S, Felli L, Plateroti AM, Perdicchi A, Contestabile MT, Recupero SM. Spectral domain optical coherence tomography evidence of retinal nerve fibre layer and ganglion cell loss in adult patients with neurofibromatosis type 1. Retina. 2016 Jan;36(1):75-81.

13 Abdolrahimzadeh S, Felli L, Piraino DC, Mollo R, Calvieri S, Recupero SM. Retinal microvascular abnormalities overlying choroidal nodules in neurofibromatosis type 1. BMC Ophthalmol. 2014 Nov;14(1):146

14 Abdolrahimzadeh S, Parisi F, Abdolrahimzadeh B, Cruciani F. Unusual choroidal vessels in neurofibromatosis type 1 observed with near-infrared reflectance and spectral domain optical coherence tomography. Acta Ophthalmol. 2016 Dec;94(8):e815-6.

15 Gu S, Glaug N, Cnaan A, Packer RJ, Avery RA. Ganglion cell layer-inner plexiform layer thickness and vision loss in young children with optic pathway gliomas. Invest Ophthalmol Vis Sci. 2014 Mar;55(3):1402-8.

16 Vallabh NA, Sahni JN, Parkes CK, Czanner G, Heimann H, Damato B. Near-infrared reflectance and autofluorescence imaging characteristics of choroidal nevi. Eye (Lond). 2016 Dec;30(12):1593-7.

17 Happle R. A rule concerning the segmental manifestation of autosomal dominant skin disorders. Review of clinical examples providing evidence for dichotomous types of severity. Arch Dermatol. 1997 Dec;133(12):1505-9.

18 Tinschert S, Naumann I, Stegmann E, Buske A, Kaufmann D, Thiel G, et al. Segmental neurofibromatosis is caused by somatic mutation of the neurofibromatosis type 1 (NF1) gene. Eur J Hum Genet. 2000 Jun;8(6):455-9.

19 Ruggieri M, Pavone P, Polizzi A, Di Pietro M, Scuderi A, Gabriele A, et al. Ophthalmological manifestations in segmental neurofibromatosis type 1. Br J Ophthalmol. 2004 Nov;88(11):1429-33.

20 Ruggieri M, Huson SM. The clinical and diagnostic implications of mosaicism in the neurofibromatoses. Neurology. 2001 Jun;56(11):1433-43.

21 Abdolrahimzadeh S, Piraino DC, Plateroti R, Scuderi G, Recupero SM. Ocular alterations in a rare case of segmental neurofibromatosis type 1 with a non-classified mutational variant of the NF1 gene. Ophthalmic Genet. 2016 Jun;37(2):214-6.

22 Cassiman C, Casteels I, Jacob J, Plasschaert E, Brems H, Dubron K, et al. Choroidal abnormalities in café-aulait syndromes: a new differential diagnostic tool? Clin Genet. 2017 Apr;91(4):529-35.

23 Arej N, Fadlallah A, Chelala E. Choroidal tubercoloma as a presenting sign of tuberculosis. Int Med Case Rep J 2016;9:365-68 (eCollection 2016).

24 Machida S, Tanaka M, Murai K, Takahashi T, Tazawa Y. Choroidal circulatory disturbance in ocular sarcoidosis without the appearance of retinal lesions or loss of visual function. Jpn J Ophthalmol. $2004 \mathrm{Jul}-$ Aug;48(4):392-6. 


\section{Case Reports in Ophthalmology}

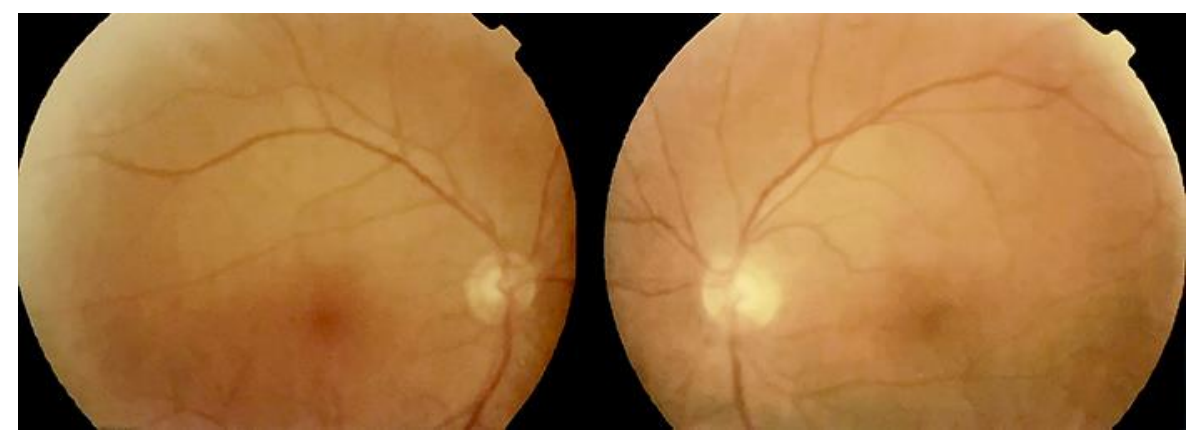

Fig. 1. Fundus photographs of the right and left eye where no remarkable alterations are visible.

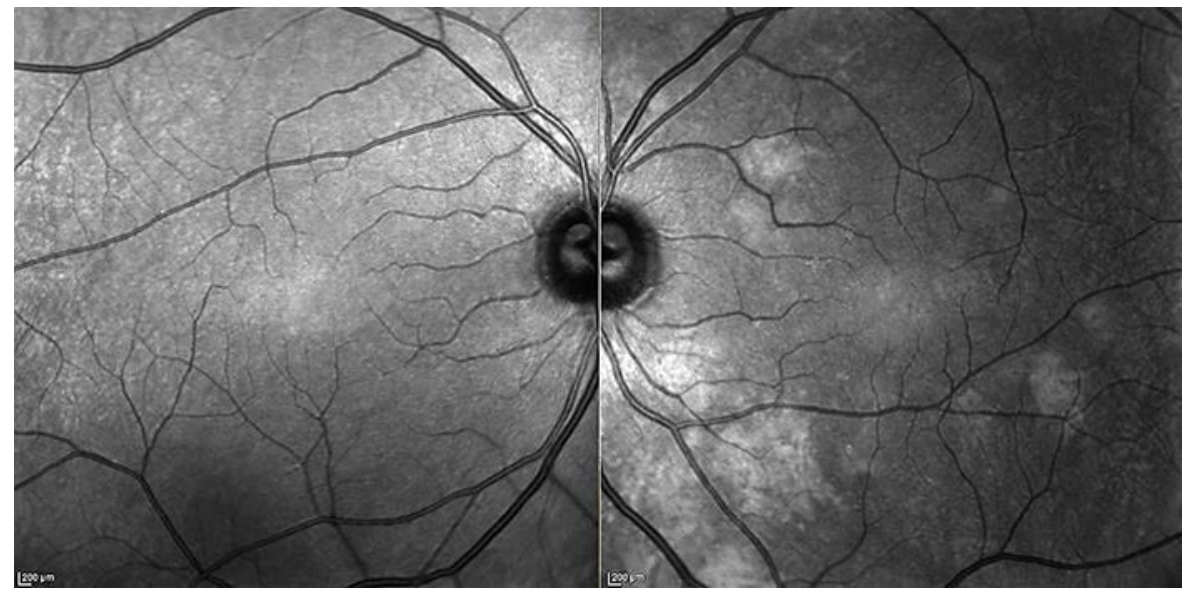

Fig. 2. Near-infrared images. The right eye does not show any alterations. The left eye shows rounded hyperreflective bright areas and irregular patchy hyperreflectivity of the posterior pole. 


\section{Case Reports in Ophthalmology}

\begin{tabular}{l|l}
\hline Case Rep Ophthalmol 2020;11:167-173 \\
\hline DOI: 10.1159/000507428 & $\begin{array}{l}\text { @ 2020 The Author(s). Published by S. Karger AG, Basel } \\
\text { www.karger.com/cop }\end{array}$ \\
\hline
\end{tabular}

Abdolrahimzadeh et al.: Indolent Choroidal Alterations

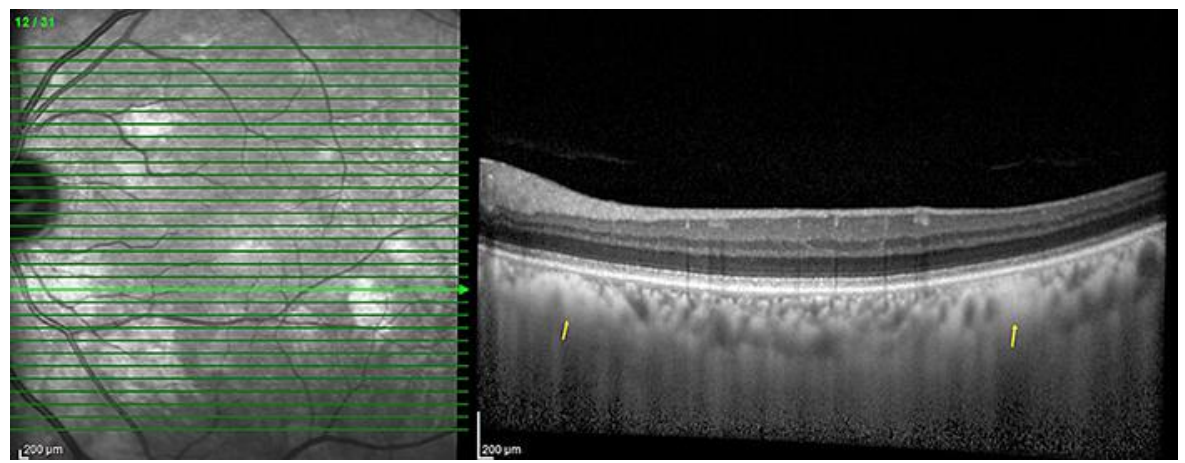

Fig. 3. Enhanced depth imaging spectral-domain optical coherence tomography images. The raster scan line on the near-infrared image on the left shows dome-shaped and placoid hyperreflective nodules typical of NF1 in the cross-sectional image.
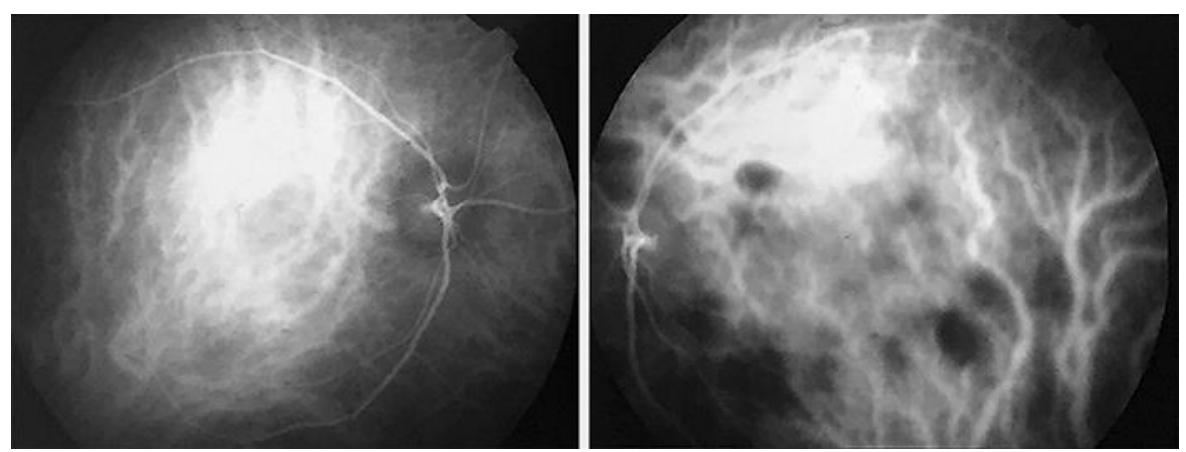

Fig. 4. ICG-A images in the late phase. The left eye shows multiple hypofluorescent areas. 